Revista Bioética

\title{
PESQUISA
}

\section{Avaliação do clima ético nos serviços de saúde: revisão sistemática}

Taís Carpes Lanes ${ }^{1}$, Ana Carolina de Souza Magnago ${ }^{1}$, Thais Costa Schutz ${ }^{1}$, Alessandra Suptitz Carneiro ${ }^{1}$, Bruna Xavier Morais ${ }^{1}$, Graziele de Lima Dalmolin ${ }^{1}$

1. Programa de Pós-Graduação em Enfermagem, Departamento de Enfermagem, Universidade Federal de Santa Maria, Santa Maria/RS, Brasil.

\section{Resumo}

O clima ético refere-se à perceptibilidade dos profissionais de saúde quanto ao tratamento ético das questões do trabalho. Este estudo buscou identificar as evidências científicas das produções que utilizaram o instrumento Hospital Ethical Climate Survey na avaliação do clima ético. Trata-se de revisão sistemática realizada nas bases de dados Scopus, PubMed e Medline, sendo selecionados 33 artigos. Evidenciou-se que o clima ético foi associado às questões de saúde do trabalhador e avaliado como de moderado a positivo pelos profissionais de saúde. O clima ético foi classificado como positivo para os fatores "pares", "pacientes" e "gestão", e como negativo para os fatores "médicos" e "hospital". Compreende-se a importância da avaliação do clima ético para a sustentabilidade e melhorias do ambiente de trabalho. Neste caso, o Hospital Ethical Climate Survey se apresentou válido e fidedigno ao ser aplicado em serviços de saúde.

Palavras-chave: Ética. Serviços de saúde. Enfermagem.

\section{Resumen}

\section{Evaluación del clima ético en servicios de salud: revisión sistemática}

El clima ético se refiere a la percepción de los profesionales de la salud sobre el tratamiento ético de las cuestiones laborales. Este estudio buscó identificar evidencias científicas de producciones que utilizaron el instrumento Hospital Ethical Climate Survey en la evaluación del clima ético. Se trata de una revisión sistemática realizada en las bases de datos Scopus, PubMed y Medline, con 33 artículos seleccionados. Se demostró que el clima ético estaba asociado a problemas de salud en el trabajo. En la evaluación por factores, el clima ético se clasificó como positivo para los factores "pares", "pacientes" y "gestión", y como negativo para los factores "médicos" y "hospital". Se concluyó que es importante evaluar el clima ético para garantizar la sostenibilidad y mejorar el clima laboral. En este caso, la aplicación del Hospital Ethical Climate Survey en los servicios de salud resultó válida y confiable. Palabras clave: Ética. Servicios de salud. Enfermería.

\begin{abstract}
Evaluation of ethical climate in health services: a systematic review

Ethical climate refers to the perceptions shared by health professionals of how ethical issues should be handled in their organization. This study aimed to identify scientific evidence of studies that used the Hospital Ethical Climate Survey in ethical climate assessment. This is a systematic review of 33 articles selected from Scopus, PubMed and Medline databases. Ethical climate was associated with workers' health issues and it was evaluated as moderate to positive by health professionals. The topic was classified as positive for the "peers," "patients" and "managers" factors, and negative for "physicians" and "hospital" factors. The review concluded that assessing the ethical climate is important for improving the work environment and that the Hospital Ethical Climate Survey is a valid and reliable instrument for such evaluation.
\end{abstract}

Keywords: Ethics. Health services. Nursing.

Declaram não haver conflito de interesse. 
O clima ético pode ser definido como a percepção de profissionais de saúde sobre o tratamento ético de questões assistenciais e das relações em ambiente de trabalho ${ }^{1,2}$. Essas questões referem-se a problemas no cuidado, diagnóstico e tratamento de pacientes, institucionalmente e entre equipes de saúde ${ }^{3}$. O clima ético é influenciado pelo comportamento organizacional e costuma ser positivo quando o ambiente favorece o exercício da autonomia e a inclusão de todos os envolvidos no cuidado, permitindo-lhes discutir e participar da tomada de decisões ${ }^{1}$.

Esse indicador vem sendo avaliado em serviços hospitalares e não hospitalares ${ }^{1,2}$. Entre os instrumentos utilizados estão o Ethical Climate Questionnaire, para serviços, indústria e comércio 2; o Ethical Decision-Making Climate Questionnaire, para unidades de terapia intensiva ${ }^{4}$; e o Hospital Ethical Climate Survey (Hecs), para áreas hospitalares ${ }^{5-7}$.

O Hecs foi desenvolvido em 1998 em Chicago, nos Estados Unidos, para avaliar a percepção de enfermeiros sobre o clima ético, e foi validado em estudo com 360 profissionais de dois hospitais de cuidados agudos em uma cidade do Oeste americano ${ }^{1}$. O instrumento é composto por cinco fatores: "pacientes", "médicos", "pares" (colegas), "hospital" (gestão do hospital) e "gestão" (gestão da unidade) ${ }^{1}$. Cada fator avalia se as relações interpessoais podem auxiliar ou dificultar a tomada ética de decisões, considerando todos os envolvidos na situação-problema ${ }^{1,8}$.

Para cada fator, o clima ético pode ser classificado como negativo, moderado ou positivo, a depender da qualidade das relações e do quão ética será a deliberação entre as partes envolvidas ${ }^{1,8,9}$. A avaliação do clima ético pode indicar intenção de rotatividade, insatisfação com o trabalho ${ }^{10}$, sofrimento do trabalhador ${ }^{11}$ e estresse moral ${ }^{12}$, além de maior tendência a cometer erros médicos ${ }^{13}$. Esses indicadores podem constranger moralmente o trabalhador, e por isso é necessário identificá-los.

Avaliando o clima ético é possível reconhecer elementos que fortalecem ou fragilizam a atuação ética e moral do trabalhador, o que se reflete na qualidade e na segurança do cuidado prestado ao paciente. Ademais, a partir de tal avaliação é possível planejar ações para melhorar o ambiente de trabalho e facilitar a tomada compartilhada de decisão com base nos preceitos das instituições e na deontologia. Considerando esses benefícios, o objetivo deste trabalho foi examinar as evidências científicas de pesquisas que utilizaram o instrumento Hecs na avaliação do clima ético.

\section{Método}

Trata-se de revisão sistemática dividida em seis etapas: 1) formulação da pergunta de revisão; 2 ) estabelecimento dos critérios de seleção e definição das bases de dados; 3 ) construção e preenchimento de formulário para registro e extração dos dados coletados; 4) avaliação crítica dos estudos primários; 5) análise e síntese descritiva dos resultados da revisão; e 6) síntese do conhecimento ${ }^{14}$.

$\mathrm{Na}$ primeira etapa, conforme a estratégia Picot - população (P), intervenção (I), comparação (C), desfecho (O) e tempo $(T)^{14}$-, delimitou-se a seguinte questão norteadora: "quais são as evidências científicas sobre a avaliação do clima ético publicadas na literatura a partir da utilização do instrumento Hecs?" Na segunda etapa, estabeleceram-se os critérios de seleção e as bases de dados pesquisadas - Scopus, PubMed e Medline Complete. Foram incluídos artigos originais, completos, publicados em inglês, português ou espanhol e que utilizassem o Hecs na abordagem do clima ético com profissionais de serviços de saúde.

$\mathrm{Na}$ base Scopus, utilizou-se a estratégia de busca "ethical climate and nursing and questionnaire or instrument or scale or validation", com 54 estudos encontrados; na PubMed, "ethical climate and nursing or nurse", com 90 publicações; e na Medline, "ethical climate and hospital ethical climate and nursing or nurse", com 87 estudos encontrados.

A busca nas bases de dados e a análise dos estudos foram realizadas em maio de 2019 , de forma duplo-independente pela autora da pesquisa e por uma bolsista de iniciação científica previamente capacitada. Foram eliminados cinco estudos pois não estavam em inglês, português ou espanhol, restando 226 publicações, que foram submetidas ao processo de seleção conforme a Figura 1.

$\mathrm{Na}$ terceira etapa, os 33 artigos selecionados foram avaliados quanto à qualidade dos dados e sua relação com o problema de pesquisa. Para a análise, foi elaborada tabela de extração de dados, que continha as seguintes informações: autoria, base de dados, área do estudo, país e ano de publicação, periódico, idioma, objetivos, método, resumo, temática (se o artigo contempla o objeto deste estudo) e principais resultados e conclusões. 
Figura 1. Fluxograma do processo de seleção duplo-independente dos artigos incluídos na revisão

Revisor A

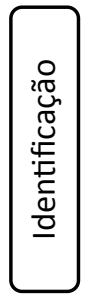

Artigos encontrados na busca

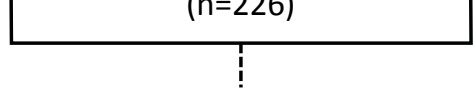

Artigos duplicados excluídos

Artigos selecionados para leitura do

党<smiles></smiles>

Artigos excluídos após leitura do

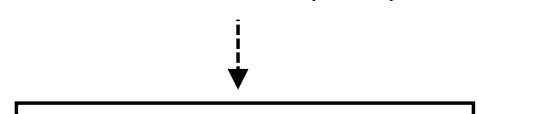

Artigos selecionados para

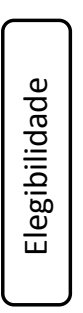

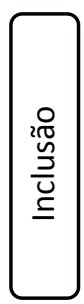

$(n=226)$

$$
(n=125)
$$

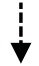
título e resumo $(n=101)$ título e resumo $(n=53)$ leitura na íntegra $(n=48)$

Revisor B

Artigos encontrados na busca $(n=226)$

Artigos duplicados excluídos $(n=125)$

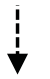

Artigos selecionados para leitura do título e resumo $(n=101)$

Artigos excluídos após leitura do título e resumo $(n=55)$

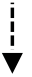

Artigos selecionados para leitura na íntegra $(n=46)$

\section{6 artigos selecionados} por ambos

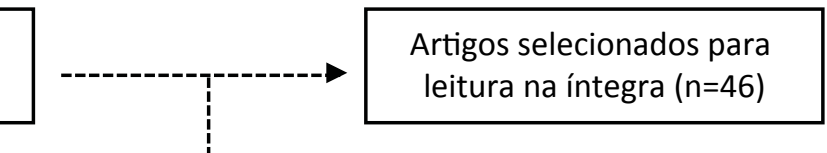

2 discordantes

2 artigos inseridos por um 3 o revisor

48 artigos para análise

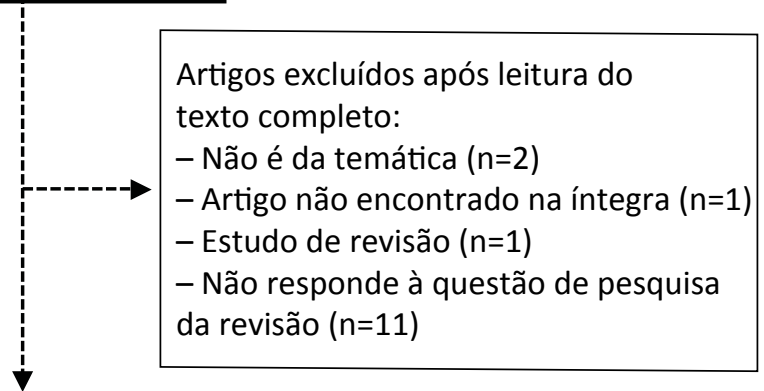

Estudos incluídos para análise $(n=33)$

A quarta etapa compreendeu a avaliação crítica dos estudos primários por meio de sistema de classificação de evidências. Diferentes classificações poderiam ser utilizadas, a depender do tipo de questão de pesquisa dos estudos primários selecionados. 0 método escolhido foi o de classificação em sete níveis: 1 ) revisão sistemática ou metanálise; 2) ensaio clínico randomizado controlado; 3) ensaios clínicos sem randomização; 4) estudos de coorte e de caso-controle; 5) revisão sistemática de estudos descritivos e qualitativos; 6) estudo descritivo ou qualitativo; e 7) opinião de autoridades e/ou relatório de comitês de especialistas ${ }^{15}$. 
A quinta etapa compreendeu análise e síntese descritiva dos resultados da revisão. Essas informações possibilitam analisar individualmente os estudos e comparar os dados. A extração tem como intuito encontrar resultados que respondam à pergunta de revisão, enfatizando as diferenças e similaridades entre os estudos selecionados, sem inferências do revisor.

Na sexta etapa, de síntese do conhecimento, são apresentados os resultados, as conclusões e limitações dos estudos, juntamente com impressões e reflexões dos autores. Cabe destacar que a presente investigação manteve a autoria e a fidedignidade dos artigos incluídos nesta revisão.

\section{Resultados}

Predominaram estudos realizados na América do Norte $(30,3 \% ; n=10)$, especificamente nos Estados Unidos (27,3\%; $n=9)^{1,3,10,11,16-20}$, entre 2014 e 2015 (36,4\%; $n=12)^{13,16-18,21-28}$, e publicados no periódico Nursing Ethics (33,3\%; $\mathrm{n}=11)^{8,9,12,13,19,25,27,29-32}$, avaliado pela Qualis Periódicos como A1 e com fator de impacto 2,597. Como campo de estudo, predominou o cenário hospitalar (84,8\%; $n=28)^{1,3,5-9,11-13,16-21,23,24,26,29-37, ~}$ principalmente unidades de cuidado agudo/crítico $(24,2 \% ; n=8)^{1,11,16,18,20,29,32,37}$. Enfermeiros participaram de todos os estudos 1,3,5-13,16-37, mas algumas pesquisas abrangeram também médicos ${ }^{6,11,17,30,37}$, auxiliares de enfermagem ${ }^{6,22,27,30}$, assistentes sociais ${ }^{10,17}$ e farmacêuticos ${ }^{17}$.

O Quadro 1 apresenta a identificação, o delineamento, os níveis de evidência, as variáveis associadas ao clima ético e os principais resultados dos estudos que compõem a amostra. Predominou o delineamento transversal de estudo (90,9\%; $n=30)$, com nível 6 de evidência.

Quadro 1. Quadro sinóptico dos artigos selecionados

\begin{tabular}{|c|c|c|c|c|}
\hline Identificação & $\begin{array}{l}\text { Delineamento } \\
\text { do estudo }\end{array}$ & $\begin{array}{l}\text { Nível de } \\
\text { evidência }\end{array}$ & $\begin{array}{l}\text { Variáveis } \\
\text { associadas ao } \\
\text { clima ético }\end{array}$ & Principais resultados \\
\hline Olson; $1998^{1}$ & Metodológico & 6 & Não apresenta & $\begin{array}{l}\text { O Hecs apresentou validade e confiabilidade } \\
\text { para medir o clima ético. }\end{array}$ \\
\hline $\begin{array}{l}\text { Bahcecik, Oztürk; } \\
2003^{5}\end{array}$ & Transversal & 6 & \begin{tabular}{|l|} 
Dados \\
sociodemográficos
\end{tabular} & $\begin{array}{l}\text { O clima ético apresentou associação com } \\
\text { tempo de experiência e idade do profissional. }\end{array}$ \\
\hline Hart; $2005^{3}$ & Transversal & 6 & $\begin{array}{l}\text { Intenção de } \\
\text { rotatividade } \\
\text { Intenção de deixar } \\
\text { a enfermagem } \\
\end{array}$ & $\begin{array}{l}\text { Os enfermeiros que perceberam o clima } \\
\text { ético como positivo relataram maior } \\
\text { intenção de permanecer no setor. } \\
\text { Relação entre clima ético e intenção de } \\
\text { deixar a enfermagem. }\end{array}$ \\
\hline $\begin{array}{l}\text { Ulrich e } \\
\text { colaboradores; } 2007^{10}\end{array}$ & Transversal & 6 & $\begin{array}{l}\text { Satisfação no } \\
\text { trabalho } \\
\text { Intenção de } \\
\text { rotatividade } \\
\text { Estresse ético }\end{array}$ & $\begin{array}{l}\text { Os participantes menos propensos a } \\
\text { rotatividade e com baixo nível de estresse } \\
\text { ético avaliaram o clima ético mais } \\
\text { positivamente. } \\
\text { Clima ético positivo aumentou a satisfação } \\
\text { no trabalho e reduziu as intenções de } \\
\text { rotatividade. }\end{array}$ \\
\hline $\begin{array}{l}\text { Hamric, Blackhall; } \\
2007^{11}\end{array}$ & Transversal & 6 & $\begin{array}{l}\text { Sofrimento moral } \\
\text { Dados laborais }\end{array}$ & $\begin{array}{l}\text { Enfermeiros com alto nível de sofrimento } \\
\text { moral avaliaram o clima ético mais } \\
\text { negativamente. } \\
\text { Enfermeiros avaliaram o clima ético mais } \\
\text { negativamente do que os médicos. }\end{array}$ \\
\hline $\begin{array}{l}\text { Pauly e } \\
\text { colaboradores; } 2009^{29} \\
\end{array}$ & Transversal & 6 & Sofrimento moral & $\begin{array}{l}\text { Quanto mais positivo o clima ético, menores } \\
\text { os níveis de sofrimento moral. }\end{array}$ \\
\hline $\begin{array}{l}\text { Lützén e } \\
\text { colaboradores; } 2010^{12}\end{array}$ & Transversal & 6 & Estresse moral & $\begin{array}{l}\text { A percepção negativa do clima ético } \\
\text { aumentou o estresse moral. }\end{array}$ \\
\hline $\begin{array}{l}\text { Silén e colaboradores; } \\
2011^{33}\end{array}$ & Transversal & 6 & Sofrimento moral & $\begin{array}{l}\text { O clima ético positivo está associado a } \\
\text { menor frequência de sofrimento moral. }\end{array}$ \\
\hline $\begin{array}{l}\text { Joolaee e } \\
\text { colaboradores; } 2013^{34}\end{array}$ & Transversal & 6 & $\begin{array}{l}\text { Satisfação no } \\
\text { trabalho }\end{array}$ & $\begin{array}{l}\text { Quanto mais positiva a percepção do clima } \\
\text { ético, maior é o nível de satisfação dos } \\
\text { enfermeiros. }\end{array}$ \\
\hline
\end{tabular}


Quadro 1. Continuação

\begin{tabular}{|c|c|c|c|c|}
\hline Identificação & $\begin{array}{l}\text { Delineamento } \\
\text { do estudo }\end{array}$ & $\begin{array}{l}\text { Nível de } \\
\text { evidência }\end{array}$ & $\begin{array}{l}\text { Variáveis } \\
\text { associadas ao } \\
\text { clima ético }\end{array}$ & Principais resultados \\
\hline $\begin{array}{l}\text { Claeys e } \\
\text { colaboradores; } 2013^{35}\end{array}$ & $\begin{array}{l}\text { Metodológico } \\
\text { e transversal }\end{array}$ & 6 & Não apresenta & $\begin{array}{l}\text { Clima ético mostrou-se positivo nas unidades. } \\
\text { A pontuação mais baixa foi para o fator } \\
\text { "hospital", e a mais alta foi para o fator "pares". }\end{array}$ \\
\hline Han; $2014^{21}$ & Transversal & 6 & $\begin{array}{l}\text { Intenção de } \\
\text { rotatividade }\end{array}$ & $\begin{array}{l}\text { Quanto mais positivo o clima ético, menor a } \\
\text { intenção de rotatividade. }\end{array}$ \\
\hline Hwang, Park; $2014^{13}$ & Transversal & 6 & $\begin{array}{l}\text { Dados laborais } \\
\text { Intenção de } \\
\text { rotatividade } \\
\text { Experiência com } \\
\text { erro médico }\end{array}$ & $\begin{array}{l}\text { Foram constatadas diferenças significativas } \\
\text { entre clima ético e anos de experiência de } \\
\text { enfermagem e ensino. } \\
\text { Os enfermeiros com percepção positiva do } \\
\text { clima ético apresentaram menor intenção } \\
\text { de rotatividade e menor probabilidade de } \\
\text { cometer erros médicos. }\end{array}$ \\
\hline $\begin{array}{l}\text { Suhonen e } \\
\text { colaboradores; } 2014^{22}\end{array}$ & Transversal & 6 & $\begin{array}{l}\text { Cuidados de } \\
\text { enfermagem } \\
\text { individualizados }\end{array}$ & $\begin{array}{l}\text { Os enfermeiros que avaliaram o clima ético } \\
\text { mais positivamente foram mais propensos } \\
\text { a perceber que os cuidados prestados eram } \\
\text { mais individualizados. }\end{array}$ \\
\hline $\begin{array}{l}\text { Sauerland e } \\
\text { colaboradores; } 2014^{16}\end{array}$ & Misto & 6 & $\begin{array}{l}\text { Sofrimento moral } \\
\text { Resíduo moral }\end{array}$ & $\begin{array}{l}\text { Os enfermeiros que relataram maiores níveis } \\
\text { de sofrimento moral avaliaram o clima ético } \\
\text { mais negativamente. }\end{array}$ \\
\hline $\begin{array}{l}\text { Khalesi e } \\
\text { colaboradores; } 2014^{23}\end{array}$ & Transversal & 6 & Não apresenta & $\begin{array}{l}\text { A maior média de clima ético foi para o fator } \\
\text { "gestão", e a menor para o fator "médicos". } \\
\text { O Hecs apresentou validade e confiabilidade } \\
\text { para medir o clima ético. }\end{array}$ \\
\hline $\begin{array}{l}\text { Ghorbani e } \\
\text { colaboradores; } 2014^{24}\end{array}$ & Transversal & 6 & $\begin{array}{l}\text { Dados } \\
\text { sociodemográficos }\end{array}$ & $\begin{array}{l}\text { Dados sociodemográficos não } \\
\text { influenciaram a percepção dos } \\
\text { enfermeiros em relação ao clima ético. }\end{array}$ \\
\hline $\begin{array}{l}\text { Whitehead e } \\
\text { colaboradores; } 2015^{17}\end{array}$ & Transversal & 6 & $\begin{array}{l}\text { Sofrimento moral } \\
\text { Dados laborais }\end{array}$ & $\begin{array}{l}\text { As percepções mais positivas para o clima } \\
\text { ético estavam associadas aos menores níveis } \\
\text { de sofrimento moral. } \\
\text { Os médicos avaliaram o clima ético mais } \\
\text { positivamente do que os enfermeiros. }\end{array}$ \\
\hline $\begin{array}{l}\text { Numminen e } \\
\text { colaboradores; } 2015^{25}\end{array}$ & Transversal & 6 & $\begin{array}{l}\text { Competência } \\
\text { profissional } \\
\text { autoavaliada } \\
\text { Intenções de } \\
\text { rotatividade } \\
\text { Satisfação no } \\
\text { trabalho }\end{array}$ & $\begin{array}{l}\text { Os enfermeiros com nível de competência } \\
\text { mais elevado, satisfeitos com o trabalho e } \\
\text { que nunca planejaram mudar de emprego } \\
\text { apresentaram percepção mais positiva do } \\
\text { clima ético. }\end{array}$ \\
\hline $\begin{array}{l}\text { Jahantigh e } \\
\text { colaboradores; } 2015^{26}\end{array}$ & Transversal & 6 & $\begin{array}{l}\text { Dados } \\
\text { sociodemográficos } \\
\text { Clima ético } \\
\text { desejado }\end{array}$ & $\begin{array}{l}\text { Relação significativa da idade e do ambiente } \\
\text { de trabalho com o clima ético. } \\
\text { Diferença significativa entre a percepção do } \\
\text { enfermeiro sobre o clima ético e o escore } \\
\text { médio desejado do clima ético. }\end{array}$ \\
\hline $\begin{array}{l}\text { Suhonen e } \\
\text { colaboradores; } 2015^{27}\end{array}$ & Transversal & 6 & Não apresenta & $\begin{array}{l}\text { O clima ético em geral foi positivo, com } \\
\text { maior pontuação para o fator "pares", e } \\
\text { menor pontuação para o fator "médicos". }\end{array}$ \\
\hline $\begin{array}{l}\text { Sauerland e } \\
\text { colaboradores; } 2015^{18} \\
\end{array}$ & Transversal & 6 & Sofrimento moral & $\begin{array}{l}\text { Relação inversa significativa entre } \\
\text { sofrimento moral e clima ético. }\end{array}$ \\
\hline $\begin{array}{l}\text { Numminen e } \\
\text { colaboradores; } \\
2015^{28}\end{array}$ & Transversal & 6 & $\begin{array}{l}\text { Ambiente de } \\
\text { prática } \\
\text { Intenção de } \\
\text { rotatividade }\end{array}$ & $\begin{array}{l}\text { Enfermeiros satisfeitos com a qualidade } \\
\text { dos cuidados avaliam mais positivamente o } \\
\text { clima ético. } \\
\text { Enfermeiros com intenções de rotatividade } \\
\text { avaliam o ambiente de prática e o clima } \\
\text { ético menos positivamente. }\end{array}$ \\
\hline
\end{tabular}


Quadro 1. Continuação

\begin{tabular}{|c|c|c|c|c|}
\hline Identificação & $\begin{array}{l}\text { Delineamento } \\
\text { do estudo }\end{array}$ & $\begin{array}{l}\text { Nível de } \\
\text { evidência }\end{array}$ & $\begin{array}{l}\text { Variáveis } \\
\text { associadas ao } \\
\text { clima ético }\end{array}$ & Principais resultados \\
\hline $\begin{array}{l}\text { Jahantigh, Zare, } \\
\text { Shahrakipour; } 2016^{36}\end{array}$ & Transversal & 6 & $\begin{array}{l}\text { Dados } \\
\text { sociodemográficos } \\
\text { Comportamento } \\
\text { ético }\end{array}$ & $\begin{array}{l}\text { As relações dos dados sociodemográficos e } \\
\text { do comportamento ético com o clima ético } \\
\text { não foram significativas. }\end{array}$ \\
\hline $\begin{array}{l}\text { Bartholdson e } \\
\text { colaboradores; } 2016^{30}\end{array}$ & Transversal & 6 & Dados laborais & $\begin{array}{l}\text { Enfermeiros avaliaram o clima ético } \\
\text { positivamente com menor frequência do que } \\
\text { os médicos. }\end{array}$ \\
\hline $\begin{array}{l}\text { Boer e colaboradores; } \\
2016^{37}\end{array}$ & Longitudinal & 4 & $\begin{array}{l}\text { Dados laborais } \\
\text { Sofrimento moral }\end{array}$ & $\begin{array}{l}\text { Enfermeiros avaliaram o clima ético mais } \\
\text { negativamente do que os médicos. } \\
\text { O clima ético positivo ajuda enfermeiros e } \\
\text { médicos a lidar melhor com o sofrimento moral. }\end{array}$ \\
\hline Jang, Oh; $2019^{31}$ & Transversal & 6 & $\begin{array}{l}\text { Satisfação no } \\
\text { trabalho } \\
\text { Liderança ética }\end{array}$ & $\begin{array}{l}\text { A satisfação com o trabalho foi positivamente } \\
\text { correlacionada com o clima ético. } \\
\text { Não houve associação significativa entre } \\
\text { clima ético e liderança ética. }\end{array}$ \\
\hline $\begin{array}{l}\text { Özden e } \\
\text { colaboradores; } 2019^{9}\end{array}$ & Transversal & 6 & $\begin{array}{l}\text { Liderança ética } \\
\text { Satisfação no } \\
\text { trabalho } \\
\text { Dados } \\
\text { sociodemográficos } \\
\text { e laborais }\end{array}$ & $\begin{array}{l}\text { Correlação positiva e significativa entre } \\
\text { liderança ética, clima ético e satisfação } \\
\text { no trabalho. } \\
\text { Diferença estatisticamente significativa entre } \\
\text { clima ético e carga horária, tipo, condições e } \\
\text { satisfação com o trabalho. } \\
\text { Os enfermeiros com mais tempo na } \\
\text { instituição apresentaram maiores escores } \\
\text { para liderança e clima ético. } \\
\text { Os enfermeiros mais satisfeitos com a } \\
\text { profissão e com o relacionamento com } \\
\text { seus colegas percebem o clima ético como } \\
\text { mais positivo. }\end{array}$ \\
\hline $\begin{array}{l}\text { Asgari e } \\
\text { colaboradores; } 2019^{32}\end{array}$ & Transversal & 6 & $\begin{array}{l}\text { Satisfação no } \\
\text { trabalho }\end{array}$ & $\begin{array}{l}\text { Relação significativa entre clima ético e } \\
\text { satisfação no trabalho. }\end{array}$ \\
\hline $\begin{array}{l}\text { Lemmenes e } \\
\text { colaboradores; } 2018^{19}\end{array}$ & Transversal & 6 & $\begin{array}{l}\text { Dados } \\
\text { sociodemográficos } \\
\text { e laborais }\end{array}$ & $\begin{array}{l}\text { Enfermeiros com menos de } 30 \text { anos de idade } \\
\text { avaliaram o clima ético mais positivamente } \\
\text { do que enfermeiros com mais de } 30 \text { anos. } \\
\text { Relação significativa entre clima ético e } \\
\text { especialidades: enfermeiros de cuidados } \\
\text { críticos adultos tiveram escores médios } \\
\text { totais mais altos. }\end{array}$ \\
\hline $\begin{array}{l}\text { Constantina, } \\
\text { Papastavrou, } \\
\text { Charalambous; } 2019^{8}\end{array}$ & Transversal & 6 & Dados laborais & $\begin{array}{l}\text { Enfermeiros com graduação avaliaram o } \\
\text { clima ético mais positivamente do que } \\
\text { enfermeiros com pós-graduação. }\end{array}$ \\
\hline $\begin{array}{l}\text { Altaker, } \\
\text { Howie-Esquivel, } \\
\text { Cataldo; } 2018^{20}\end{array}$ & Transversal & 6 & $\begin{array}{l}\text { Sofrimento moral } \\
\text { Empoderamento } \\
\text { psicológico }\end{array}$ & $\begin{array}{l}\text { Enfermeiros que avaliaram o clima ético } \\
\text { mais positivamente apresentaram níveis } \\
\text { mais baixos de sofrimento moral. } \\
\text { Correlação positiva entre Hecs e } \\
\text { Psychological Empowerment Instrument. }\end{array}$ \\
\hline $\begin{array}{l}\text { Charalambous e } \\
\text { colaboradores; } 2018^{7}\end{array}$ & Metodológica & 6 & Não apresentou & $\begin{array}{l}\text { O Hecs apresentou validade e confiabilidade } \\
\text { para medir o clima ético. }\end{array}$ \\
\hline $\begin{array}{l}\text { Pergert, Bartholdson, } \\
\text { Sandeberg; } 2019^{6}\end{array}$ & Transversal & 6 & Dados laborais & $\begin{array}{l}\text { Médicos avaliaram o clima ético mais } \\
\text { positivamente do que enfermeiros e } \\
\text { auxiliares de enfermagem. }\end{array}$ \\
\hline
\end{tabular}

Hecs: Hospital Ethical Climate Survey 
Evidenciaram-se associações do clima ético com outras variáveis $(84,8 \% ; n=28)^{3,5,6,8-13,16-22,24-26,28-34,36,37}$. Na maior parte dos estudos, o clima ético foi associado a sofrimento moral, avaliado pela Moral Distress Scale (24,2\%; $n=8)$ 11,16-18,20,29,33,37; satisfação no trabaIho, de acordo com os instrumentos Job Satisfaction Scale $^{10}$, Minnesota Job Satisfaction Questionnaire ${ }^{34}$, Minnesota Satisfaction Questionnaire ${ }^{31}$, Minnesota Satisfaction Scale ${ }^{9}$ e Brayfield and Rothe Job
Satisfaction Index ${ }^{32}$ (15,2\%; n=5); e intenção de rotatividade, avaliadas por meio da Anticipated Turnover Scale ${ }^{3}$ e do Turnover Intention ${ }^{21}(6,1 \% ; n=2)$. Além disso, foram utilizadas questões sobre satisfação no trabalho ${ }^{25}$ e intenção de rotatividade ${ }^{10,13,25,28}$ elaboradas pelos próprios autores dos estudos (15,2\%; $\mathrm{n}=5$ ). Na Tabela 1 estão dispostos os valores da média e do desvio-padrão utilizados na classificação geral e por fatores do Hecs.

Tabela 1. Classificação geral e por fatores do Hospital Ethical Climate Survey

\begin{tabular}{|c|c|c|c|c|c|c|}
\hline \multirow{3}{*}{ Identificação* } & \multicolumn{5}{|c|}{ Classificação do clima ético/Hecs por fatores } & \multirow{2}{*}{$\begin{array}{c}\text { Classificação } \\
\text { geral do clima } \\
\text { ético/Hecs }\end{array}$} \\
\hline & Pacientes & Pares & Médicos & Gestão & Hospital & \\
\hline & Média (DP) & Média (DP) & Média (DP) & Média (DP) & Média (DP) & Média (DP) \\
\hline $\begin{array}{l}\text { Ulrich e } \\
\text { colaboradores; } 2007^{10}\end{array}$ & - & - & - & - & - & $97,3(14,4)$ \\
\hline $\begin{array}{l}\text { Pauly e } \\
\text { colaboradores; } 2009^{29}\end{array}$ & $3,71(0,573)$ & $4,12(0,617)$ & $3,35(0,741)$ & $3,40(1,071)$ & $3,11(0,747)$ & $3,48(0,612)$ \\
\hline $\begin{array}{l}\text { Silén e colaboradores; } \\
2011^{33}\end{array}$ & - & - & - & - & - & $95,00(* *)$ \\
\hline $\begin{array}{l}\text { Joolaee e } \\
\text { colaboradores; } 2013^{34}\end{array}$ & - & - & - & - & - & $3,36(0,69)$ \\
\hline $\begin{array}{l}\text { Claeys e } \\
\text { colaboradores; } 2013^{35}\end{array}$ & $3,90(0,49)$ & $4,15(0,49)$ & $3,75(0,59)$ & $3,85(0,77)$ & $3,64(0,59)$ & $3,85(0,46)$ \\
\hline Han; $2014^{21}$ & $3,35(0,40)$ & $3,61(0,50)$ & $2,76(0,60)$ & $3,28(0,60)$ & $2,78(0,50)$ & $3,11(* *)$ \\
\hline Hwang, Park; $2014^{13}$ & $3,60(0,60)$ & $3,7(0,70)$ & $3,0(0,80)$ & $3,8(0,80)$ & $3,3(0,60)$ & $3,5(0,60)$ \\
\hline $\begin{array}{l}\text { Suhonen e } \\
\text { colaboradores; } 2014^{22}\end{array}$ & - & - & - & - & - & $3,85(0,56)$ \\
\hline $\begin{array}{l}\text { Sauerland e } \\
\text { colaboradores; } 2014^{16}\end{array}$ & - & - & - & - & - & $94,39(18,3)$ \\
\hline $\begin{array}{l}\text { Khalesi e } \\
\text { colaboradores; } 2014^{23}\end{array}$ & $2,80(0,64)$ & $2,90(0,65)$ & $2,46(0,69)$ & $3,04(0,66)$ & $2,61(0,72)$ & $2,75(0,58)$ \\
\hline $\begin{array}{l}\text { Ghorbani e } \\
\text { colaboradores; } 2014^{24}\end{array}$ & $\begin{array}{l}3,94(0,64)^{+} \\
4,01(0,65)^{++}\end{array}$ & $\begin{array}{c}4,12(0,5)^{+} \\
4,05(0,64)^{++}\end{array}$ & $\begin{array}{l}3,31(0,76)^{+} \\
3,46(0,73)^{++}\end{array}$ & $\begin{array}{l}4,23(0,77)^{+} \\
4,24(0,76)^{++}\end{array}$ & $\begin{array}{l}3,37(0,81)^{+} \\
3,48(0,88)^{++}\end{array}$ & $\begin{array}{l}3,76(0,54)^{+} \\
3,82(0,61)^{++}\end{array}$ \\
\hline $\begin{array}{l}\text { Whitehead e } \\
\text { colaboradores; } 2015^{17}\end{array}$ & - & - & - & - & - & $58,2(11,1)$ \\
\hline $\begin{array}{l}\text { Numminen e } \\
\text { colaboradores; } 2015^{25}\end{array}$ & $4,10(0,52)$ & $4,33(0,54)$ & $3,74(0,58)$ & $3,50(0,91)$ & $3,54(0,64)$ & $3,84(0,45)$ \\
\hline $\begin{array}{l}\text { Jahantigh e } \\
\text { colaboradores; } 2015^{26}\end{array}$ & $14,70(2,76)$ & $15,42(2,66)$ & $20,98(4,40)$ & $22,07(3,93)$ & $21,6(4,15)$ & $94,78(15,35)$ \\
\hline $\begin{array}{l}\text { Suhonen e } \\
\text { colaboradores; } 2015^{27}\end{array}$ & $3,96(0,54)$ & $4,29(0,55)$ & $3,58(0,71)$ & $3,94(0,87)$ & $3,64(0,67)$ & $3,85(0,56)$ \\
\hline $\begin{array}{l}\text { Sauerland e } \\
\text { colaboradores; } 2015^{18}\end{array}$ & - & - & - & - & - & $96,6(17,77)$ \\
\hline $\begin{array}{l}\text { Jahantigh, Zare, } \\
\text { Shahrakipour; } 2016^{36}\end{array}$ & - & - & - & - & - & $94,78(15,35)$ \\
\hline $\begin{array}{l}\text { Boer e colaboradores; } \\
2016^{37}\end{array}$ & - & - & - & - & - & $3,86(0,46)$ \\
\hline Jang, Oh; $2019^{31}$ & $3,69(0,40)$ & $4,01(0,48)$ & $3,61(0,57)$ & $3,98(0,56)$ & $3,46(0,47)$ & $3,59(0,41)$ \\
\hline $\begin{array}{l}\text { Özden e } \\
\text { colaboradores; } 2019^{9}\end{array}$ & - & - & - & - & - & $92,62(17,0)$ \\
\hline
\end{tabular}


Tabela 1. Continuação

\begin{tabular}{|c|c|c|c|c|c|c|}
\hline \multirow{3}{*}{ Identificação* } & \multicolumn{5}{|c|}{ Classificação do clima ético/Hecs por fatores } & \multirow{2}{*}{$\begin{array}{c}\text { Classificação } \\
\text { geral do clima } \\
\text { ético/Hecs }\end{array}$} \\
\hline & Pacientes & Pares & Médicos & Gestão & Hospital & \\
\hline & Média (DP) & Média (DP) & Média (DP) & Média (DP) & Média (DP) & Média (DP) \\
\hline $\begin{array}{l}\text { Asgari e } \\
\text { colaboradores; } 2019^{32}\end{array}$ & $3,29(* *)$ & $3,72(* *)$ & $1,58(* *)$ & $3,8(* *)$ & $2,61(* *)$ & $3,51(0,53)$ \\
\hline $\begin{array}{l}\text { Lemmenes e } \\
\text { colaboradores; } 2018^{19}\end{array}$ & $3,60(0,60)$ & $3,94(0,65)$ & $2,93(0,81)$ & $3,04(1,06)$ & $2,97(0,74)$ & $3,22(0,65)$ \\
\hline $\begin{array}{l}\text { Constantina, } \\
\text { Papastavrou, } \\
\text { Charalambous; } 2019^{8} \\
\end{array}$ & $3,74(0,69)$ & $3,99(0,64)$ & $3,06(0,79)$ & $3,88(0,92)$ & $3,4(0,72)$ & $3,58(0,62)$ \\
\hline $\begin{array}{l}\text { Altaker, } \\
\text { Howie-Esquivel, } \\
\text { Cataldo; } 2018^{20}\end{array}$ & - & - & - & - & - & $3,9(0,5)$ \\
\hline
\end{tabular}

*Nove artigos ${ }^{1,3,5-7,11,12,28,30}$ não apresentaram média e desvio-padrão da classificação geral do clima ético; ** não apresenta desvio-padrão; ${ }^{+}$hospital $A ;{ }^{++}$hospital B; - não apresentaram classificação por fatores; DP: desvio-padrão; Hecs: Hospital Ethical Climate Survey

Como mostra a Tabela 1, entre os estudos que avaliaram o clima ético por fatores, $84,6 \%(n=11)$ apresentaram escores mais altos para os fatores "pacientes", "pares" e "gestão", classificados como positivos, e escores mais baixos para "hospital" e "médicos" 8,13,19,21,23,24,27,29,31,32,35, classificados como negativos. O clima ético avaliado a partir do Hecs foi de moderado a positivo. Em apenas um estudo a classificação foi negativa ${ }^{23}$.

Notou-se que os estudos avaliaram o Hecs de duas maneiras: pela soma e pela média dos valores do instrumento. Oito $(24,2 \%)^{9,10,16-18,26,33,36}$ o avaliaram a partir da soma dos itens; $16(48,5 \%)^{8,13,19-25,27,29,31,32,34,35,37}$ pela média geral do instrumento; $12(36,4 \%)^{8,13,19,21,23-25,27,29,31,32,35}$ pela média por fatores; e $5(15,2 \%)^{8,16,18,23,25}$ pela média por itens referente aos valores assinalados em escala Likert.

Alguns estudos ${ }^{20,35}$ descreveram, a partir da análise da média, o valor para classificar o clima ético como positivo (acima de 3,5 ) ou negativo (abaixo de 3,5). Outros ${ }^{9,16,18,24,32}$ classificaram o clima ético pela soma de pontos do instrumento, sendo 130 considerado o valor máximo, e 26 o mínimo. 0 clima ético foi avaliado como positivo quando acima de 78 pontos, e negativo quando abaixo desse valor ${ }^{26,36}$.

Os artigos não descritos na Tabela 1 realizaram somente pesquisas de associação/correlação entre clima ético e outras variáveis ${ }^{3,11,12,28,30}$, eram estudos de validação ${ }^{1,7}$ ou utilizaram porcentagens para avaliar o clima ético de forma geral e/ou por itens do instrumento Hecs ${ }^{5,30}$. O Hecs também foi utilizado em forma reduzida, passando de 26 para $14^{6}$ e $17^{30}$ itens em unidades hemato-oncológicas; para $15^{11} \mathrm{e}$
$25^{37}$ itens em unidades de cuidado agudo/crítico; e para $16^{17}$ itens em setores hospitalares.

A confiabilidade geral do instrumento variou de 0,85 a 0,95 e foi avaliada por meio do alfa de Cronbach (63,6\%; $n=21$ ) 1,3,5,7-12,18-20,22-24,27,31-33,35,37. Já a confiabilidade por fatores foi calculada em $21,2 \%(n=7)$ dos artigos, nos quais o fator "pares" variou de 0,70 a 0,88 ; "pacientes", de 0,58 a 0,85 ; "gestão", de 0,73 a 0,93; "hospital", de 0,58 a 0,83; e "médicos", de 0,71 a $0,89^{1,7,8,19,23,27,35}$.

\section{Discussão}

Quanto à caracterização dos estudos, prevaleceram produções científicas realizadas nos Estados Unidos, em cenário hospitalar, principalmente em unidades críticas, o que se justifica pelo fato de o Hecs ter sido desenvolvido em Chicago e em áreas hospitalares. A escolha dos estudos para avaliar o clima ético em unidades críticas talvez se deva ao modo operante de trabalho, voltado a cuidados mais específicos e complexos, o que pode gerar mais dilemas e conflitos éticos a serem resolvidos, demandando tomadas de decisão e deliberação moral ${ }^{16}$.

Ademais, em alguns estudos, o clima ético avaliado pelo Hecs apresentou associação/correlação com sofrimento moral, intenção de rotatividade e satisfação no trabalho. Evidências científicas demonstram que o clima ético positivo está, de um lado, ligado à redução de fatores que geram sofrimento moral e estresse no ambiente laboral, intenção de rotatividade e abandono da profissão e, 
de outro, ao fortalecimento da equipe e à satisfação com o trabalho 10,12,13,21,25,32-34,37.

O sofrimento moral foi a variável que mais apresentou associações significativas com o clima ético ${ }^{16,20,32}$. Isso ocorre, em especial, pela dificuldade dos profissionais em tomar decisões de maneira compartilhada ${ }^{18}$. Além disso, a insegurança dos colegas em relação ao cuidado dos pacientes e à escassez de profissionais para demandas de alta intensidade causa sofrimento e resulta em ambiente pouco colaborativo, aumentando a percepção negativa do clima ético ${ }^{16}$.

Em unidades pediátricas e neonatais, por exemplo, onde profissionais prestam assistência a crianças que precisam de cuidados de alta complexidade, observam-se situações que favorecem o desenvolvimento de sofrimento moral, associadas à experiência de presenciar o sofrimento de pacientes e familiares ${ }^{16}$. Nesse contexto, uma relação equilibrada da equipe pode tornar o trabalho mais leve, aliviando tensões durante a assistência ${ }^{29}$.

Em geral, a avaliação do clima ético foi de moderada a positiva ${ }^{13,19,21,25,27,29,31,32,35,36}$. No entanto, questões que analisaram o relacionamento entre enfermeiros e médicos apresentaram as menores médias ${ }^{13,21,24,26}$. Os estudos identificaram dificuldades na interação entre essas categorias profissionais, possivelmente por falta de comunicação e de discussão sobre assuntos relacionados a diagnóstico e tratamento ${ }^{38}$. A enfermagem, por estar mais próxima dos pacientes, poderia oferecer observações e elementos importantes para definir o tratamento, porém muitas vezes os enfermeiros são afastados da tomada de decisão ${ }^{38}$.

As dificuldades nas relações podem estar associadas a percepções diferentes sobre o que seria o ambiente ideal de trabalho. É o que sugerem estudos em unidades oncológicas e de cuidados críticos, que, ao comparar percepções de diferentes categorias profissionais, detectaram avaliação mais positiva do clima ético entre médicos ${ }^{6,11}$. Isso pode estar relacionado às diferentes responsabilidades $\mathrm{e}$ competências de cada profissão dentro das unidades de trabalho ${ }^{30,37}$. Nesse contexto, o enfermeiro teria olhar mais crítico sobre o processo laboral por estar inserido nos serviços de saúde por período mais extenso, buscando compreender o que é a situação de conflito e deliberar sobre ela com a equipe pela qual é responsável ${ }^{30}$.

Diferentemente da relação entre enfermeiros e médicos, estudos apresentaram percepções positivas entre os pares, indicando relacionamento de apoio e cooperação ${ }^{13,19,21,25,27,29,31,35}$. Essas relações estão associadas à prática de exercer liderança e ao uso da ferramenta de escuta entre colegas, que contribuem com a interação necessária para realizar as atividades assistenciais de forma homogênea ${ }^{8,25}$. Logo, relações saudáveis auxiliam na tomada de decisões e no enfretamento de dilemas, além de possibilitar a prestação de cuidados com mais segurança para os pacientes ${ }^{8,30}$. Nessa perspectiva, a maior parte dos estudos apresentou clima ético positivo para o fator "pacientes", o que evidencia relação de respeito mútuo ${ }^{13,19,25,27,29,31,35}$. Quando o profissional respeita a autonomia do paciente no tratamento e sua inserção no cuidado, a interação melhora e há mais confiança nas relações ${ }^{30}$.

Entretanto, a percepção dos profissionais foi negativa em relação ao fator "hospital". A falta de apoio e abertura para questionamentos influenciou negativamente o clima ético ${ }^{13,21,23,25,29,31,35}$. A gestão teria que auxiliar o desenvolvimento e a manutenção de ambiente saudável, promovendo espaços de discussão com os trabalhadores ${ }^{26}$. Assim, seria importante que gestores liderassem o enfrentamento dos problemas que acometem as instituições, estabelecendo normas éticas para orientar os profissionais sobre como agir diante de dilemas ${ }^{23,31}$.

A gestão da unidade, que na maioria dos estudos recebeu avaliação positiva, precisa se empenhar em melhorar o trabalho de equipe, investindo no aperfeiçoamento do local e transmitindo aos trabaIhadores sua vontade de beneficiar o clima organizacional e a assistência ${ }^{39}$. A valorização das categorias profissionais permite diversificar o trabalho e construir relacionamento de respeito, que estimula o desempenho da equipe ${ }^{34}$.

Em relação às análises do instrumento, o estudo original de validação do $\mathrm{Hecs}^{1}$ avaliou o clima ético por meio da média das pontuações, porém não houve normalização do instrumento, já que alguns estudos utilizaram a soma 9,10,16-18,26,33,36. Essa situação pode dificultar a análise pela falta de padronização. No entanto, o Hecs foi utilizado de forma reduzida em unidades críticas em outros países ${ }^{6,11,30,37}$, o que o torna instrumento facilmente adaptável para aplicação em diferentes locais.

Dessa maneira, evidenciou-se que o Hecs é válido e fidedigno para medir o clima ético em meio hospitalar, visto que a confiabilidade do instrumento ficou acima de 0,70 nos estudos que realizaram o cálculo de alfa de Cronbach 1,3,5,7-12,18-20,22-24,27,31-33,35,37. Essa medida justifica a aplicação com profissionais de unidades de saúde - não somente enfermeiros ${ }^{1,3,5-13,16-37}$, mas também médicos ${ }^{6,11,17,30,37}$, 
auxiliares de enfermagem ${ }^{6,22,27,30}$, assistentes sociais ${ }^{10,17}$ e farmacêuticos ${ }^{17}$.

\section{Considerações finais}

O clima ético, na maior parte dos estudos, mostrou-se associado a questões relativas à saúde do trabalhador, como sofrimento moral, satisfação no trabalho e intenção de rotatividade. Além disso, a classificação do clima ético, a partir do Hecs, foi de moderada a positiva, tendo maiores escores para os fatores "pares", "pacientes" e "gestão", classificados como positivos, e menores escores para os fatores "médicos" e "hospital", classificados como negativos.

O Hecs, no geral, se mostrou válido e fidedigno para medir o clima ético. É necessário, contudo, adaptar e validar o instrumento para o Brasil, no intuito de avaliar o clima ético em serviços de saúde com diferentes categorias profissionais, além de realizar pesquisas que investiguem associações entre clima ético e outras questões da saúde do trabalhador, como burnout, estresse e assédio moral, considerando ações de intervenção. Nessa perspectiva, deve-se buscar melhorar o clima ético e a qualidade da assistência à saúde, reduzindo problemas psíquicos entre os trabalhadores.

\section{Referências}

1. Olson LL. Hospital nurses' perceptions of the ethical climate of their work setting. J Nurs Scholarsh [Internet]. 1998 [acesso 17 abr 2018];30(4):345-9. DOI: 10.1111/j.1547-5069.1998.tb01331.x

2. Victor B, Cullen JB. The organizational bases of ethical work climates. Adm Sci Q [Internet]. 1988 [acesso 17 abr 2018];33(1):101-25. DOI: 10.2307/2392857

3. Hart SE. Hospital ethical climates and registered nurses' turnover intentions. J Nurs Scholarsh [Internet]. 2005 [acesso 12 maio 2018];37(2):173-7. DOI: 10.1111/j.1547-5069.2005.00030.x

4. Benoit DD, Jensen HI, Malmgren J, Metaxa V, Reyners AK, Darmon M et al. Outcome in patients perceived as receiving excessive care across different ethical climates: a prospective study in 68 intensive care units in Europe and the USA. Intensive Care Med [Internet]. 2018 [acesso 12 maio 2018];44:1039-49. DOI: 10.1007/s00134-018-5231-8

5. Bahcecik N, Oztürk H. The hospital ethical climate survey in Turkey. Jonas Healthc Law Ethics Regul [Internet]. 2003 [acesso 15 maio 2018];5(4):94-9. Disponível: https://bit.ly/32QxeZU

6. Pergert $P$, Bartholdson C, Sandeberg $M$. The ethical climate in paediatric oncology: a national cross-sectional survey of health-care personnel. Psychooncology [Internet]. 2019 [acesso 10 maio 2019];28(4):735-41. DOI: 10.1002/pon.5009

7. Charalambous A, Cloconi C, Papastavrou E, Theodoula A. Psychometric properties of the Hospital Ethical Climate Survey: a cross-sectional study in Greek and Cypriot cancer care settings. J Nurs Meas [Internet]. 2018 [acesso 20 maio 2018];26(2):237-48. DOI: 10.1891/1061-3749.26.2.237

8. Constantina C, Papastavrou E, Charalambous A. Cancer nurses' perceptions of ethical climate in Greece and Cyprus. Nurs Ethics [Internet]. 2019 [acesso 15 maio 2018];26(6):1805-21. DOI: $10.1177 / 0969733018769358$

9. Özden D, Arslan GG, Ertuğrul B, Karakaya S. The effect of nurses' ethical leadership and ethical climate perceptions on job satisfaction. Nurs Ethics [Internet]. 2019 [acesso 15 maio 2020];26(4):1211-25. DOI: 10.1177/0969733017736924

10. Ulrich C, O'Donnell P, Taylor C, Farrar A, Danis M, Grady C. Ethical climate, ethics stress, and the job satisfaction of nurses and social workers in the United States. Soc Sci Med [Internet]. 2007 [acesso 12 maio 2018];65(8):1708-19. DOI: 10.1016/j.socscimed.2007.05.050

11. Hamric $A B$, Blackhall $L$. Nurse-physician perspectives on the care of dying patients in intensive care units: collaboration, moral distress, and ethical climate. Crit Care Med [Internet]. 2007 [acesso 12 maio 2018];35(2):422-9. DOI: 10.1097/01.CCM.0000254722.50608.2D

12. Lützén $\mathrm{K}$, Blom T, Ewalds-Kvist $\mathrm{B}$, Winch $\mathrm{S}$. Moral stress, moral climate and moral sensitivity among psychiatric professionals. Nurs Ethics [Internet]. 2010 [acesso 6 maio 2018];17(2):213-24. DOI: 10.1177/0969733009351951

13. Hwang JI, Park HA. Nurses' perception of ethical climate, medical error experience and intent-to-leave. Nurs Ethics [Internet]. 2014 [acesso 6 maio 2018];21(1):28-42. DOI: $10.1177 / 0969733013486797$

14. Paula CC, Padoin SMM, Galvão CM. Revisão integrativa como ferramenta para tomada de decisão na prática em saúde. In: Lacerda MR, Costenaro RGS, organizadores. Metodologias da pesquisa para a enfermagem e saúde: da teoria à prática. Porto Alegre: Moriá; 2016. p. 51-76.

15. Melnyk BM, Fineout-Overholt E. Evidence-based practice in nursing and healthcare: a guide to best practice. 2 a ed. Philadelphia: Wolters Kluwer; 2011.

16. Sauerland J, Marotta K, Peinemann MA, Berndt A, Robichaux C. Assessing and addressing moral distress and ethical climate, part I. Dimens Crit Care Nurs [Internet]. 2014 [acesso 20 abr 2018];33(4):234-45. DOI: 10.1097/DCC.0000000000000050 
17. Whitehead PB, Herbertson RK, Hamric AB, Epstein EG, Fisher JM. Moral distress among healthcare professionals: report of an institution-wide survey. J Nurs Scholarsh [Internet]. 2015 [acesso 18 abr 2018];47(2):117-25. DOI: 10.1111/jnu.12115

18. Sauerland J, Marotta K, Peinemann MA, Berndt A, Robichaux C. Assessing and addressing moral distress and ethical climate part II: neonatal and pediatric perspectives. Dimens Crit Care Nurs [Internet]. 2015 [acesso 18 abr 2018];34(1):33-46. DOI: 10.1097/DCC.0000000000000083

19. Lemmenes $D$, Valentine $P$, Gwizdalski $P$, Vincent $C$, Liao $C$. Nurses' perception of ethical climate at a large academic medical center. Nurs Ethics [Internet]. 2018 [acesso 9 maio 2018];25(6):724-33. DOI: $10.1177 / 0969733016664980$

20. Altaker KW, Howie-Esquivel J, Cataldo JK. Relationships among palliative care, ethical climate, empowerment, and moral distress in intensive care unit nurses. Am J Crit Care [Internet]. 2018 [acesso 10 maio 2019];27(4):295-302. DOI: 10.4037/ajcc2018252

21. Han SJ. Nurse turnover intention and influencing factors. Int Inf Institute [Internet]. 2014 [acesso 10 maio 2019];17(10A):5037-42. Disponível: https://bit.ly/3nsWEFa

22. Suhonen R, Stolt M, Gustafsson ML, Katajisto J, Charalambous A. The associations among the ethical climate, the professional practice environment and individualized care in care settings for older people. J Adv Nurs [Internet]. 2014 [acesso 14 maio 2018];70(6):1356-68. DOI: 10.1111/ jan.12297

23. Khalesi N, Arabloo J, Khosravizadeh O, Taghizadeh S, Heyrani A, Ebrahimian A. Psychometric properties of the Persian version of the Hospital Ethical Climate Survey. J Med Ethics Hist Med [Internet]. 2014 [acesso 20 maio 2018];7:15. Disponível: https://bit.ly/3nyyvwJ

24. Ghorbani AA, Hesamzadeh A, Khademloo M, Khalili S, Hesamzadeh S, Berger V. Public and private hospital nurses' perceptions of the ethical climate in their work settings, Sari City, 2011. Nurs Midwifery Stud [Internet]. 2014 [acesso 20 maio 2018];3(1):e12867. DOI: 10.17795/ nmsjournal12867

25. Numminen O, Leino-Kilpi $\mathrm{H}$, Isoaho $\mathrm{H}$, Meretoja R. Ethical climate and nurse competence: newly graduated nurses' perceptions. Nurs Ethics [Internet]. 2015 [acesso 5 maio 2018];22(8):845-59. DOI: $10.1177 / 0969733014557137$

26. Jahantigh M, Arbabisarjou A, Zare S, Shahrakipour M, Ghoreishinia G. Hospital's ethical climate and nurse's desired ethical climate in Ali-ebn-Abitaleb and Khatam-al-Anbia hospital of Zahedan. Pharm Lett [Internet]. 2015 [acesso 20 maio 2018];7(12):427-31. Disponível: https://bit.ly/32VFF6f

27. Suhonen R, Stolt M, Katajisto J, Charalambous A, Olson LL. Validation of the Hospital Ethical Climate Survey for older people care. Nurs Ethics [Internet]. 2015 [acesso 16 maio 2018];22(5):517-32. DOI: 10.1177/0969733014549878

28. Numminen $\mathrm{O}$, Leino-Kilpi $\mathrm{H}$, Isoaho $\mathrm{H}$, Meretoja R. Newly graduated nurses' competence and individual and organizational factors: a multivariate analysis. J Nurs Scholarsh [Internet]. 2015 [acesso 4 maio 2018];47(5):446-57. DOI: 10.1111/jnu.12153

29. Pauly B, Varcoe C, Storch J, Newton L. Registered nurses' perceptions of moral distress and ethical climate. Nurs Ethics [Internet]. 2009 [acesso 19 abr 2018];16(5):561-73. DOI: 10.1177/0969733009106649

30. Bartholdson C, Sandeberg M, Lützén K, Blomgren K, Pergert P. Healthcare professionals' perceptions of the ethical climate in paediatric cancer care. Nurs Ethics [Internet]. 2016 [acesso 20 maio 2018];23(8):877-88. DOI: 10.1177/0969733015587778

31. Jang Y, Oh Y. Impact of ethical factors on job satisfaction among Korean nurses. Nurs Ethics [Internet]. 2019 [acesso 10 maio 2019];26(4):1186-98. DOI: 10.1177/0969733017742959

32. Asgari S, Shafipour V, Taraghi Z, Yazdani-Charati J. Relationship between moral distress and ethical climate with job satisfaction in nurses. Nurs Ethics [Internet]. 2019 [acesso 20 abr 2019];26(2):346-56. DOI: 10.1177/0969733017712083

33. Silén M, Svantesson M, Kjellström S, Sidenvall B, Christensson L. Moral distress and ethical climate in a Swedish nursing context: perceptions and instrument usability. J Clin Nurs [Internet]. 2011 [acesso 20 abr 2018];20(23-24):3483-93. DOI: 10.1111/j.1365-2702.2011.03753.x

34. Joolaee S, Jalili HR, Rafii F, Hajibabaee F, Haghani H. The relationship between ethical climate at work and job satisfaction among nurses in Tehran. Indian J Med Ethics [Internet]. 2013 [acesso 12 maio 2018];10(4):238-42. DOI: 10.20529/IJME.2013.072

35. Claeys M, Faelens A, Sabbe B, Schrijvers D, Casterlé BDD, Luyten P. Psychometric properties of the Hospital Ethical Climate Survey: a cross-sectional study in a large sample of Belgian psychiatric nurses. Eur J Pers Cent Healthc [Internet]. 2013 [acesso 12 maio 2018];1(1):202-8. DOI: 10.5750/ ejpch.v1i1.652

36. Jahantigh $\mathrm{M}$, Zare $\mathrm{S}$, Shahrakipour $\mathrm{M}$. The survey of the relationship between ethical climate and ethical behavior in nurses. Pharm Chem [Internet]. 2016 [acesso 20 maio 2018];8(3):189-93. Disponível: https://bit.ly/3IELjB2

37. Boer JC, Rosmalen J, Bakker AB, Dijk M. Appropriateness of care and moral distress among neonatal intensive care unit staff: repeated measurement. Nurs Crit Care [Internet]. 2016 [acesso 13 maio 2018];21(3):e19-27. DOI: 10.1111/nicc.12206

38. Pimentel D. Relações e conflitos éticos na prática de médicos e enfermeiros. Brasília: CFM; 2017. 
39. Esmaelzadeh F, Abbaszadeh A, Borhani F, Peyrovi H. Ethical leadership and organizational climate: the experience of Iranian nurses. Iran Red Crescent Med J [Internet]. 2017 [acesso 15 maio 2018];19(4):e43554. DOI: 10.5812/ircmj.43554

\section{Participação das autoras}

Taís Carpes Lanes analisou e interpretou os dados e, com Ana Carolina de Souza Magnago, Thais Costa Schutz, Alessandra Suptitz Carneiro e Bruna Xavier Morais, redigiu o artigo. Graziele de Lima Dalmolin orientou e revisou o manuscrito.

\section{Correspondência}

Taís Carpes Lanes - Rua João Goulart, 401, casa 2, fundos, Camobi CEP 97105-220. Santa Maria/RS, Brasil.

Taís Carpes Lanes - Doutoranda - taislanes_rock@hotmail.com

\section{(iD) 0000-0001-9337-7875}

Ana Carolina de Souza Magnago - Graduanda - anasmagnago@gmail.com

(iD) $0000-0003-4902-4110$

Thais Costa Schutz - Mestranda - thais.schutz@hotmail.com

(iD) 0000-0003-4341-0395

Alessandra Suptitz Carneiro - Doutoranda - alessandracarneiro1994@gmail.com

(iD) 0000-0001-7506-8606

Bruna Xavier Morais - Doutoranda - bruna_morais100@hotmail.com

(iD) 0000-0002-0446-9231

Graziele de Lima Dalmolin - Doutora - grazi.dalmolin@gmail.com

(iD) 0000-0003-0985-5788

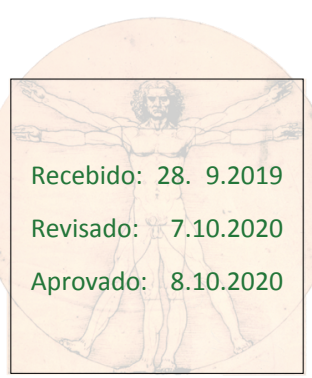

